\title{
Letter
}

\section{Cisplatin-mediated cytotoxicity through inducing CYP4A 11 expression in human renal tubular epithelial cells}

\author{
Jin $\mathrm{Li}^{1, *}$, Dao $\mathrm{Li}^{2, *}$, Chaorong Tie ${ }^{3}$, Ji Wu${ }^{2}$, Qiong $\mathrm{Wu}^{2}$ and Qixiong $\mathrm{Li}^{2}$ \\ 'Department of Laboratory, Zhongnan Hospital of Wuhan University, Wuhan 430071, China \\ ${ }^{2}$ Department of Pharmacology, City College, Wuhan University of Science and Technology, Wuhan 430083, China \\ ${ }^{3}$ Department of Stomatology, Zhongnan Hospital of Wuhan University, Wuhan 430071, China
}

(Received September 11, 2015; Accepted October 20, 2015)

\begin{abstract}
Cisplatin (CP) is a major antineoplastic drug for the treatment of solid tumors, but it has dose-dependent renal tubular toxicity. Previous studies have shown that induction of cytochrome P450 (CYP) by CP may play a role in the renal injury of CP. The aim of this study was to investigate the relationship between CP-induced toxicity and CYP4A11 expression in human renal tubular epithelial cells (HK-2). 20-Hydroxyeicosatetraenoic acid (20-HETE) is a CYP4A11 metabolite of arachidonic acid that plays an important role in renal injury. The activity of lactate dehydrogenase (LDH) was determined by spectrophotometer. CYP4A11 expression was analyzed by immunocytochemistry. CYP4A11 mRNA and protein expression were evaluated by RT-PCR and Western blot analyses. Results showed that 20-HETE $(1,10,50 \mu \mathrm{M})$, a CYP4A11 metabolite of arachidonic acid, significantly increased lactate dehydrogenase $(\mathrm{LDH})$ release in these cells. When $\mathrm{CP}\left(10^{-4} \mathrm{M}\right)$ and 20 -HETE $(1,10,50 \mu \mathrm{M})$ were co-applied to these cells, CP-induced LDH release was significantly exaggerated by 20-HETE. Furthermore, clofibrate, a CYP4A inducer, also increased LDH release in CP-treated cells. In contrast, the CYP4A inhibitor $\mathrm{N}$-Hydrocy-N'-(-4-butyl-2-methylphenyl) formamidine (HET-0016) decreased LDH release in CP-treated cells. Immunocytochemical analysis showed that CYP4A11expression was much stronger in CP$\left(10^{-4} \mathrm{M}\right)$ treated cells than that in clofibrate-treated cells. Further RT-PCR and Western blot analyses demonstrated that CYP4A11 mRNA and protein expression were significantly up-regulated in CP- $\left(10^{-4} \mathrm{M}\right)$ treated cells compared to the clofibrate group. The findings of this study indicate that $\mathrm{CP}$ is a potent inducer of CYP4A11, and it exerts its toxic functions via the induction of CYP4A11 and 20-HETE generation.
\end{abstract}

Key words: Cisplatin, CYP4A11, Expression, 20-HETE, HK-2, Cytotoxicity

\section{INTRODUCTION}

Cisplatin (CP) is a major antineoplastic drug used for the treatment of solid tumors. Its chief dose-limiting side effect is nephrotoxicity; $20 \%$ of patients receiving high-dose CP have severe renal dysfunction (Yao et al., 2007; Schrier et al., 2004; Merouani et al., 1996). CP nephrotoxicity primarily causes tubulointerstitial lesions (Vickers et al., 2004). In animal models, CP damages the proximal tubules, specifically the S3 segment of the outer medullary stripe (Portilla et al., 2006).

Although several studies have shown that reactive oxygen species (ROS) or free radicals are closely related to the nephrotoxicity induced by CP (Liu et al., 2008; Baliga et al., 1998), the detailed mechanisms of CP-in- duced renal tubular toxicity have not been fully understood. However, in the literature, CP-induced hepatotoxicity is enhanced by elevated expression of cytochrome P450 (CYP) 2E1 (Lu and Cederbaum, 2006); and it has been reported that CYP2E1 contributes to CP nephrotoxicity (Liu et al., 2002).

In humans, two isoforms of CYP4A have been identified: CYP4A11 and CYP4A22 (Bellamine et al., 2003). These two genes share $96 \%$ sequence identity and have similar intron/exon sizes and distribution. CYP4A11 is the predominant isoform expressed in human kidneys, while the level of CYP4A22 mRNAs is low (Bellamine et al., 2003). Rat CYPs CYP4A1, CYP4A2, CYP4A3, and CYP4A 8 and human CYP4A11 are known to be expressed in the kidneys and to metabolize arachidonic

Correspondence: Chaorong Tie (E-mail: ewysls@163.com)

Qixiong Li (E-mail: liqixiong336685@126.com)

*These authors equally contributed to this work. 
acid (AA) to 20-Hydroxyeicosatetraenoic acid (20-HETE) (Hoch et al., 2000). 20-HETE serves as an important regulator of vascular tone, renal blood flow, and renal tubular sodium transport (Kroetz and Zeldinm, 2002; Rahman et al., 1997).

An earlier study has shown that CP induces CYP4A1 in rat kidneys, and induction of CYP4A1 by CP may play a role in the renal injury or nephrotoxicity of CP (Nakamura et al., 1998). However, the effects of CP on the expression of CYP4A11 enzymes and 20-HETE in human renal tubular epithelial cell lines (HK-2) remain poorly understood. Therefore, the present study was designed to investigate the role of CYP4A11 expression and 20-HETE in CP-induced cytotoxicity in HK-2 cells.

\section{MATERIALS AND METHODS}

\section{Test chemicals}

CP, 20-HETE, HET-0016, and clofibrate were obtained from Sigma (St. Louis, MO, USA). Dulbecco's modified eagle medium (DMEM), ECL chemiluminescence kit and SYBR Green Real-time PCR Master Mix were purchased from Toyobo Company (Tokyo, Japan). Trizol kit was purchased from Invitrogen (Carlsbad, CA, USA). CYP4A11 monoclonal antibody and goat anti-mouse IgG were obtained from Santa Cruz (Carlsbad, CA, USA). Streptavidin peroxidase kit, LDH measurement kit, and protease inhibitor cocktail were purchased from Beijing Zhongshan Biotechnology Co. Ltd. (Beijing, China).

\section{Cell lines and cell culture conditions}

HK-2 (China Type Culture Collection; Wuhan, China) cells were maintained at $37^{\circ} \mathrm{C}$ in a humidified incubator with $5 \% \mathrm{CO}_{2} / 95 \%$ air and propagated in DMEM containing $100 \mathrm{mg} / \mathrm{dL}$ d-glucose, $10 \%$ fetal calf serum, $100 \mathrm{U} / \mathrm{mL}$ penicillin, $100 \mu \mathrm{g} / \mathrm{mL}$ streptomycin, and $2 \mathrm{mM}$ supplemental glutamine. Cells were passaged every $72 \mathrm{hr}$ by light trypsinization. Experiments were initiated by resting cells for $24 \mathrm{hr}$ in $0.5 \%$ fetal calf serum. Cells were used for experiments from passages 5-15.

\section{Cytotoxicity assay}

Cytotoxicity was measured using the LDH activity assay. Briefly, cells were plated in 96-well tissue culture plates at a density of $4 \times 10^{4}$ cells $/ \mathrm{mL}$. Cells were washed twice with phosphate-buffered saline (PBS), followed by incubation in serum-deprived medium. During this incubation period, HK-2 were treated with one of the following: effects of 20-HETE on HK-2 toxicity: 1) control: cells were treated with vehicle only, 2) 20-HETE: cells were treated with 20-HETE with the following con- centrations $(1,10,50 \mu \mathrm{M})$ for $14 \mathrm{hr}$; effects of 20-HETE on $\mathrm{LDH}$ release in the presence of $\mathrm{CP}$ in $\mathrm{HK}-2: 1)$ vehicle (dimethyl sulfoxide), 2) $\left.\mathrm{CP}\left(10^{-4} \mathrm{M}\right), 3\right) \mathrm{CP}\left(10^{-4} \mathrm{M}\right)+$ 20-HETE $(1,10,50 \mu \mathrm{M})$ for $14 \mathrm{hr}$; effects of clofibrate on LDH activity in the presence of CP in HK-2: 1) vehicle (dimethyl sulfoxide), 2) clofibrate $(50 \mu \mathrm{M}), 3) \mathrm{CP}$ $\left.\left(10^{-4} \mathrm{M}\right), 4\right) \mathrm{CP}\left(10^{-4} \mathrm{M}\right)+$ clofibrate $(50 \mu \mathrm{M})$ for $14 \mathrm{hr}$; effects of HET-0016 on LDH activity in the presence of CP in HK-2: 1) vehicle (dimethyl sulfoxide), 2) HET-0016 $\left.(1 \mu \mathrm{M}), 3) \mathrm{CP}\left(10^{-4} \mathrm{M}\right), 4\right) \mathrm{CP}\left(10^{-4} \mathrm{M}\right)+$ HET-0016 $(1 \mu \mathrm{M})$ for $14 \mathrm{hr}$. Each concentration was tested for at least five replicates from five different batches of cells. The activity of LDH was determined spectrophotometrically by measuring the oxidation rate of nicotinamide adenine dinucleotide-reduced in a thermostated cuvette at $440 \mathrm{~nm}$ after incubation of cells with Na-pyruvate, L-aspartate and L-alanin. The enzyme activity was calculated in relation to the unit volume (1) and expressed as international Units (U). The Michaelis-Menten constants were calculated in relation to the substrate concentration.

\section{CYP4A11 determined by immunocytochemistry}

HK-2 were treated with one of the following: 1) control, 2) HET-0016 (1 $\mu \mathrm{M}), 3)$ clofibrate $(50 \mu \mathrm{M}), 2) \mathrm{CP}$ $\left(10^{-4} \mathrm{M}\right)$ for $14 \mathrm{hr}$. Each concentration was tested for at least three replicates. Slides of cells were fixed in dimethylketone for $10 \mathrm{~min}$. Streptavidin peroxidase (SP) method was used. CYP4A11 monoclonal antibody (1:50) as primary antibodies was applied and incubated overnight at $4^{\circ} \mathrm{C}$ or for $60 \mathrm{~min}$ at room temperature. The slides of cells were observed and photographed through light microscope and positive cells were stained yellow brown in the cytoplasm.

\section{CYP4A11 mRNA levels determined by RT-PCR}

HK-2 were treated with one of the following: 1) solvent, 2) clofibrate (50 $\mu \mathrm{M}), 3)$ HET-0016 (1 $\mu \mathrm{M}), 4) \mathrm{CP}$ $\left(10^{-6}\right.$ to $\left.10^{-4} \mathrm{M}\right)$. Cells were treated with their respective drugs for $14 \mathrm{hr}$ before samples were collected for PCR analysis. Each concentration was tested for at least three experiments. RNA was extracted with the One Step Trizol kit. Primer sequences were as follows: CYP4A11 (sense: ATGGGTTTCTCTGTATTTAGCCC, antisense: TCTTAGCTTCTTGAGACGCAGG); $\beta$-actin (sense: GAGGGAAATCGTGCGTGAC, antisense: CTGGAAGGTGGACAGTGAG). $\beta$-actin was used as an internal control for sample normalization. cDNA steps were omitted. RTPCR reaction: reaction system was for a total volume of $25 \mu \mathrm{L}$ including SYBR Green mix $12.5 \mu \mathrm{L}$, primer (5 pmol $/ \mu \mathrm{L}) 2 \mu \mathrm{L}$, cDNA (10-fold dilution) $2.5 \mu \mathrm{L}$, $\mathrm{ddH}_{2} \mathrm{O} 8 \mu \mathrm{L}$; the conditions were as follows: $50^{\circ} \mathrm{C}$ for 
Cisplatin-induced CYP4A11expression in HK-2

$2 \mathrm{~min}, 95^{\circ} \mathrm{C}$ for $2 \mathrm{~min}, 95^{\circ} \mathrm{C}$ for $15 \mathrm{sec}$, annealing for $15 \mathrm{sec}, 72^{\circ} \mathrm{C}$ for $45 \mathrm{sec}, 72^{\circ} \mathrm{C}$ for $10 \mathrm{~min}, 40$ cycles.

\section{CYP4A11 protein levels determined by Western blot}

Cell groups and treatments were the same as in RTPCR analysis. Briefly, HK-2 were washed in phosphatebuffered saline and then lysed in $1 \mathrm{~mL}$ of $1 \%$ Nonidet P-40, 25 mM Tris- $\mathrm{HCl}, 150 \mathrm{mM} \mathrm{NaCl}, 10 \mathrm{mM}$ ethylenediamine tetraacetic acid, $\mathrm{pH} 8.0$, containing a 1:50 dilution of a protease inhibitor cocktail for $30 \mathrm{~min}$ on ice. Samples were centrifuged at $14,000 \mathrm{~g}$ for $5 \mathrm{~min}$ to pellet cell debris. Supernatant samples $(20 \mu \mathrm{g})$ were mixed with sodium dodecyl sulfate-polyacrylamide gel electrophoresis sample buffer, boiled for $5 \mathrm{~min}$, electrophoresed on a $10 \%$ sodium dodecyl sulfate polyacrylamide gel, and electroblotted onto Hybond-ECL nitrocellulose membranes. The membrane was blocked in PBS containing 5\% skimmed milk powder and $0.02 \%$ Tween 20 . To detect CYP4A11 protein, the membrane was incubated for $1 \mathrm{hr}$ at room temperature with goat polyclonal antibody against CYP4A11. After washing, the membrane was incubated with a 1:20,000 dilution of peroxidaseconjugated goat anti-mouse IgG in phosphate-buffered saline containing 1\% normal goat serum and $1 \%$ fetal calf serum. The blot was then developed using the electrochemiluminescence detection kit to produce a chemiluminescence signal, which was captured on x-ray films. Equal loading of proteins was confirmed by probing the same membrane with an antibody against $\beta$-actin.

(a)

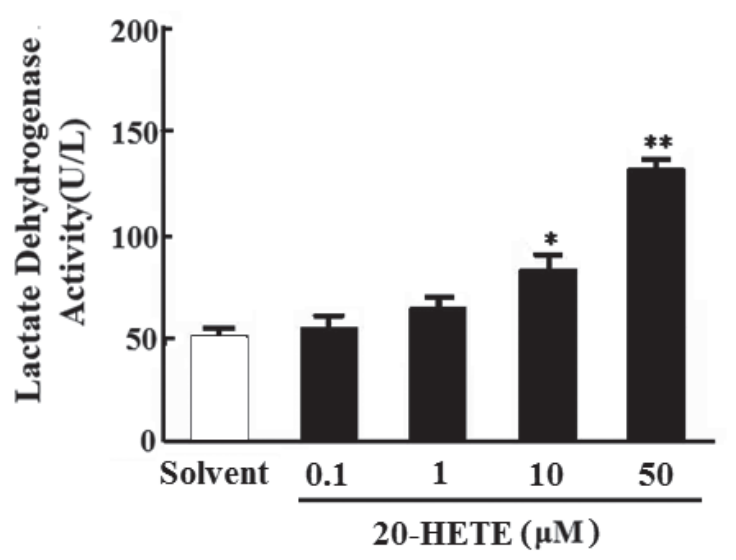

\section{Statistical analysis}

Data were expressed as mean \pm S.D. Comparisons between groups were performed by one-way ANOVA, followed by Newman-Keuls test for comparing treated with control samples. The $p$ values of $<0.05$ are considered significant. All statistical analyses were performed using SPSS software 17.0 (Released Aug. 23, 2008; Chicago, IL, USA).

\section{RESULTS}

\section{Cytotoxicity induced by $20-\mathrm{HETE}$}

HK-2 treated with 20-HETE $(10,50 \mu \mathrm{M})$ alone showed significant increase in LDH activity in comparison with the control group $(P<0.05, P<0.01)$, indicating its cytotoxicity (Fig. 1a). When CP $\left(10^{-4} \mathrm{M}\right)$ and 20-HETE $(1,10,50 \mu \mathrm{M})$ were co-applied to HK-2, CP-induced LDH activity was significantly enhanced by 20 -HETE $(P<0.05, P<0.01)$ (Fig. 1b), indicating that 20 -HETE exaggerated $\mathrm{CP}$ toxicity in these cells.

\section{Effects of clofibrate and HET-0016 on LDH activity in the presence of CP}

As shown in Fig. 2a, the HK-2 cells were incubated with $\mathrm{CP}\left(10^{-4} \mathrm{M}\right)$ and clofibrate $(50 \mu \mathrm{M})$ for $14 \mathrm{hr}$, CPinduced LDH activity was effectively increased by clofibrate as compared to the CP group $(P<0.01)$, indicating that clofibrate exaggerated CP toxicity in these cells. Fig. $2 b$ shows that in $\mathrm{CP}\left(10^{-4} \mathrm{M}\right)$ and HET-0016 $(1 \mu \mathrm{M})$ co-treated HK-2, CP-induced LDH activity was fur-

\section{(b)}

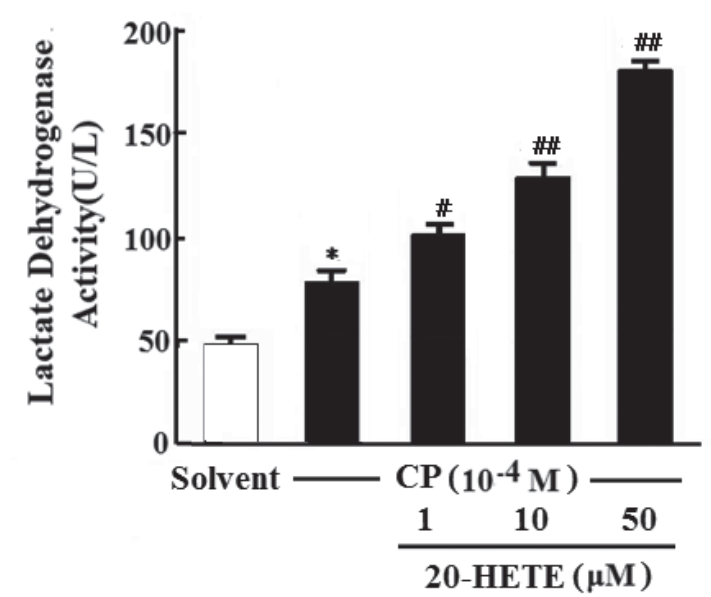

Fig. 1. Effects of 20-HETE on LDH activity in the presence of CP in HK-2. Data were expressed as mean value \pm S.D. of at least five samples derived from five separate experiments. (a) $* P<0.05, * * P<0.01$ compared with control group. (b) $* P<0.01$ compared with control group; ${ }^{P} P<0.01,{ }^{\#} P<0.01$ compared with $\mathrm{CP}$ group. 
(a)

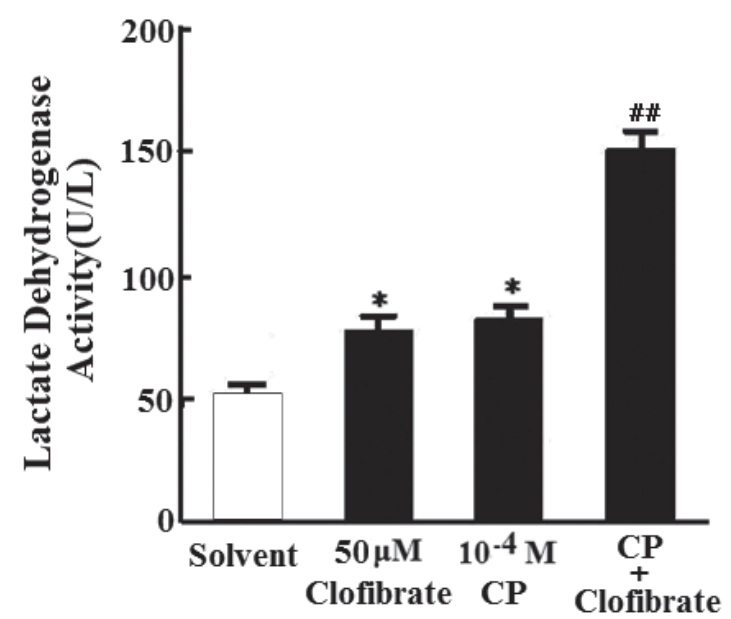

(b)

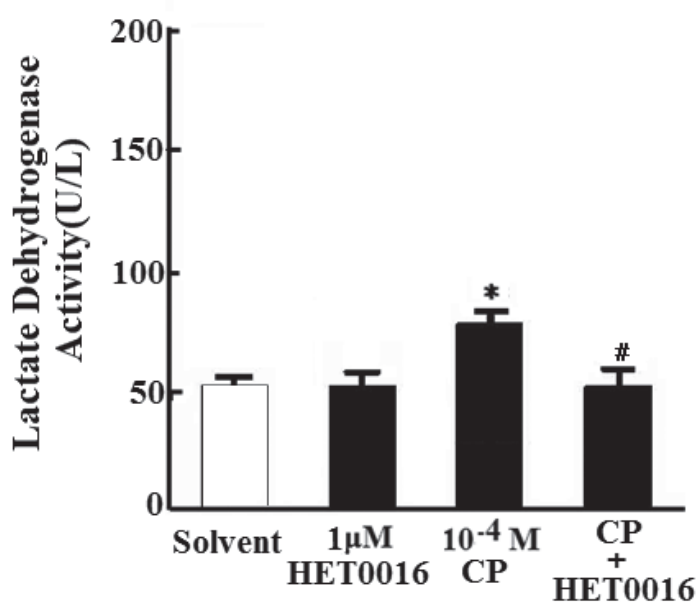

Fig. 2. Effects of clofibrate and HET-0016 on LDH activity in the presence of CP in HK-2. Data are expressed as mean value \pm S.D. of at least from five samples derived from five separate experiments. (a) clofibrate: $* P<0.05$ compared with control group; ${ }^{\#} P<0.01$ compared with CP group. (b) HET-0016: ${ }^{*} P<0.05$ compared with control group; ${ }^{*} P<0.01$ compared with CP group.

ther reversed by HET-0016 as compared to the CP group $(P<0.05)$, indicating that HET-0016 decreased CP toxicity in these cells.

\section{CYP4A11 expression determined by immunocytochemistry}

Immunocytochemical analysis demonstrated that weak CYP4A11 expression was observed in control HK-2 (Fig. 3a). CYP4A11 expression was weaker in the cells treated with HET-0016 compared to the control group (Fig. 3b). In contrast, CYP4A11 expression was significantly increased in clofibrate- $(50 \mu \mathrm{M})$ treated cells compared to the HET-0016 group (Fig. 3c). CYP4A11 expression was much stronger in $\mathrm{CP}-\left(10^{-4} \mathrm{M}\right)$ treated cells than that in clofibrate-treated cells (Fig. 3d).

\section{CYP4A11 mRNA and protein expression assay}

The results of the RT-PCR and Western blot analyses are summarized in Fig. 4. CYP4A11 mRNA (Fig. 4a) and protein (Fig. 4b) expression were significantly lower than that in the control group $(P<0.01)$ in HET-0016-treated cells. In contrast, CYP4A11 mRNA (Fig. 4a) and protein (Fig. 4b) expression were significantly up-regulated in cells treated respectively with $\mathrm{CP}\left(10^{-4} \mathrm{M}\right)$ compared to the clofibrate $(50 \mu \mathrm{M})$ group $(P<0.05)$, indicating that $\mathrm{CP}$ induced the expression of CYP4A11 mRNA and protein in $\mathrm{HK}-2$.

\section{DISCUSSION}

The anticancer drug $\mathrm{CP}$ causes acute renal failure in experimental animal models and humans (Safirstein et al., 1986; Screnci and McKeage, 1999). The nephrotoxicity of CP is well documented as the most important doselimiting factor in cancer chemotherapy. It is evident that CYP2E1 plays an important role in CP-induced cytotoxicity by serving as a site for the generation of ROS and a significant source of catalytic iron (Liu et al., 2002). A previous study has shown that induction of CYP4A1 in rat kidneys by CP may take part in renal injury by CP (Nakamura et al., 1998). Cytochrome P4504A11 in human kidneys is localized in the S2 and S3 segments of proximal tubular epithelia in the cortex and outer medulla (Lasker et al., 2000).

CYP4A11 is the predominant isoform expressed in human kidneys (Bellamine et al., 2003), and it remains unclear whether it participates in CP renal tubular injury. The role of CYP4A11 in CP-induced renal injury or potentiation of $\mathrm{CP}$ renal tubular injury has not been previously examined. In the present study, we evaluated whether CP-induced renal tubular injury is enhanced by elevated CYP4A11 expression.

Results from our study demonstrated that HK-2 treated with 20-HETE alone showed significant increase in LDH release. When CP and 20-HETE were co-applied to these cells, CP-induced LDH release was effectively increased 

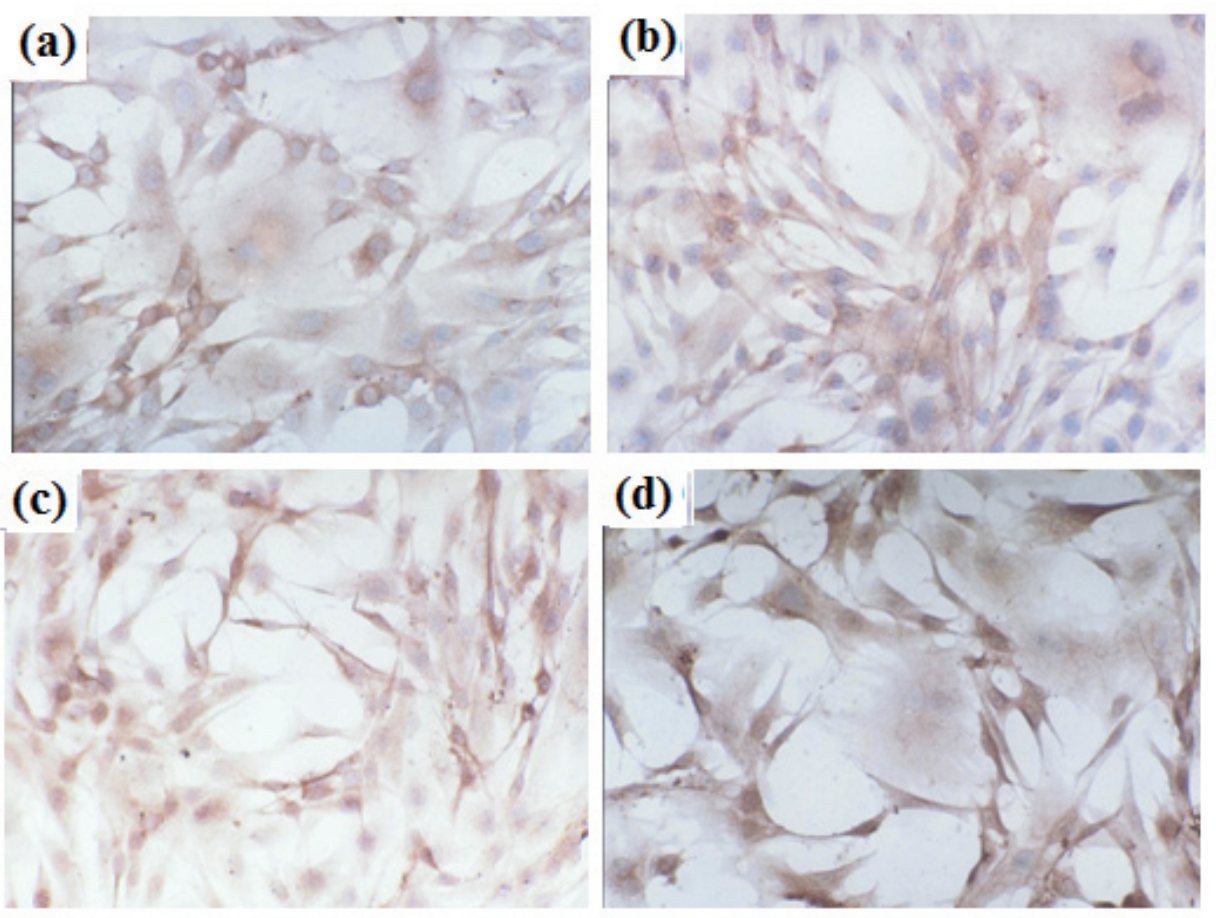

Fig. 3. Immunocytochemistry analyzed the CYP4A11 expression in HK-2 (SP, $400 \times)$. Cytoplasm was stained brown-yellow in CYP4A11 expression. (a) Control group: CYP4A11 expression was weak. (b) HET-0016 $(1 \mu \mathrm{M})$ : CYP4A11 expression was minor. (c) Clofibrate $(50 \mu \mathrm{M})$ : CYP4A11 expression was abundant. (d) CP $\left(10^{-4} \mathrm{M}\right)$ : CYP4A11 expression was more abundant.

(a)

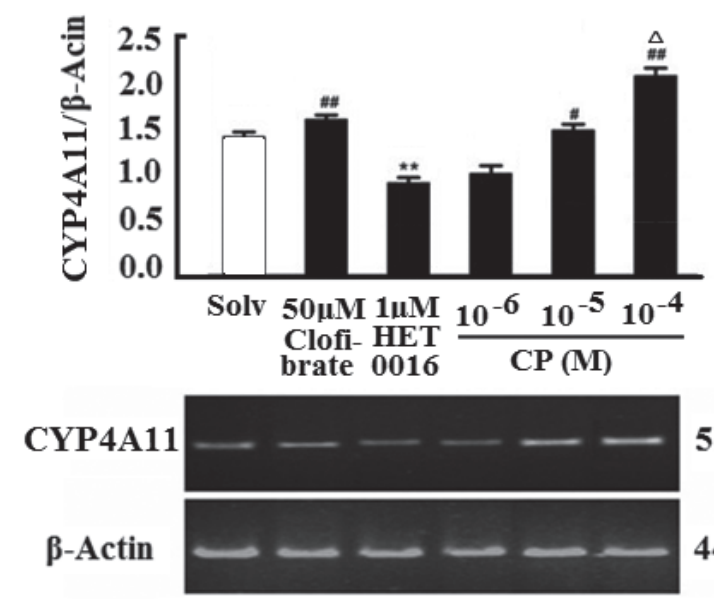

(b)

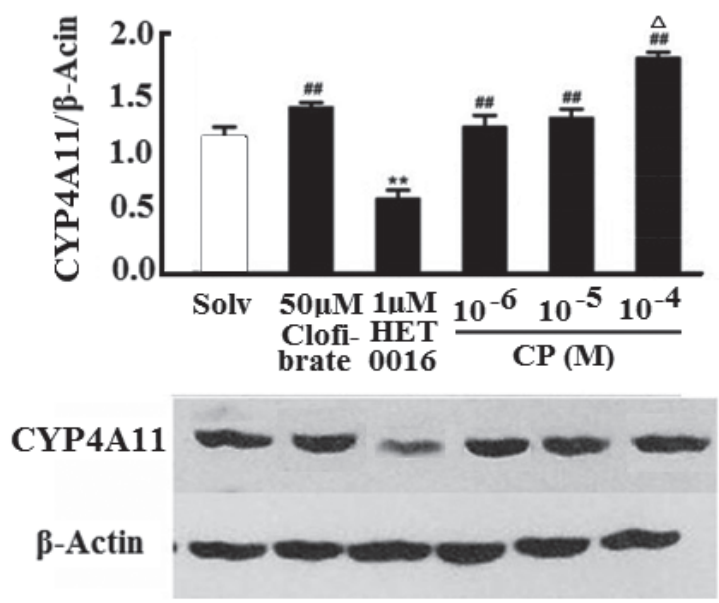

Fig. 4. PCR and Western blot analyzes of CYP4A11 mRNA and protein expression in HK-2. (a) PCR analyses demonstrated that CP $\left(10^{-6}\right.$ to $\left.10^{-4} \mathrm{M}\right)$ had distinguished up-regulation on CYP4A11 mRNA expression in HK-2. Data are expressed as mean \pm S.D. for groups of three experiments. ${ }^{*} * P<0.01$ compared with control group; ${ }^{*} P<0.05,{ }^{\#} P<0.01$ compared with HET0016 group. ${ }^{\triangle} P<0.05$ compared with clofibrate group. (b) Western blot analyses showed that $\mathrm{CP}\left(10^{-6}\right.$ to $\left.10^{-4} \mathrm{M}\right)$ had distinguished up-regulation on CYP4A11 protein expression in HK-2. Data are expressed as mean \pm S.D. for groups of three experiments. $* * P<0.01$ compared with control group; ${ }^{\#} P<0.01$ compared with HET-0016 group. ${ }^{\Delta} P<0.05$ compared with clofibrate group. 
by 20-HETE. Furthermore, the CYP4A inducer clofibrate increased LDH release in CP-treated cells. In contrast, the CYP4A inhibitor HET-0016 decreased LDH release in CP-treated cells. Immunocytochemical analysis showed that CYP4A11 expression was much stronger in CP-treated cells than in clofibrate cells. Further RT-PCR and Western blot analyses demonstrated that CYP4A11 mRNA and protein expression were significantly up-regulated in CP-treated cells compared to the clofibrate group. It has been reported that $\mathrm{CP}$ has similar effects to clofibrate and induced CYP4A1 in rat kidneys, although the effects were at a lesser extent than those of clofibrate (Nakamura et al., 1998). Both in vitro and in vivo results indicate that elevated CYP2E1 enhances CP-induced hepatotoxicity, and the mechanism may involve increased production of ROS and oxidative stress (Lu and Cederbaum, 2006). $\mathrm{CP}$-induced cell death in CYP2E1-overexpressing HepG2 cells modulation mechanism by ROS, glutathione, and thioredoxin (Lu and Cederbaum, 2007). Mechanisms of CP-mediated cytotoxicity through inducing CYP4A11 expression in HK-2 will require further study.

In conclusion, these results suggest that $\mathrm{CP}$ is a potent inducer of CYP4A11, and it exerts its toxic functions via the induction of CYP4A11 and 20-HETE generation. CPinduced CYP4A11 may represent a novel mechanism by which this drug induces cytotoxicity.

\section{ACKNOWLEDGMENTS}

This work was supported by a research grant (No.81273601) from the National Natural Science Foundation of China.

Conflict of interest---- The authors declare that there is no conflict of interest.

\section{REFERENCES}

Baker, J.R., Satarug, S., Urbenjapol, S., Edwards, R.J., Williams, D.J., Moore, M.R. and Reilly, P.E. (2002): Associations between human liver and kidney cadmium content and immunochemically detected CYP4A11 apoprotein. Biochem. Pharmacol., 63, 693-696.

Baliga, R., Zhang, Z., Baliga, M., Ueda, N. and Shah, S.V. (1998): Role of cytochrome P-450 as a source of catalytic iron in cisplatin-induced nephrotoxicity. Kidney Int., 54, 1562-1569.

Bellamine, A., Wang, Y., Waterman, M.R., Gainer, J.V.3rd., Dawson, E.P., Brownb, N.J. and Capdevila, J.H. (2003): Characterization of the CYP4A11 gene, a second CYP4A gene in humans. Arch. Biochem. Biophys., 409, 221-227.

Hoch, U., Zhang, Z., Kroetz, D.L. and Ortiz de Montellano, P.R. (2000): Structural determination of the substrate specificities and regioselectivities of the rat and human fatty acid $\omega$-hydroxylases. Arch. Biochem. Biophys., 373, 63-71.

Kroetz, D.L. and Zeldinm, D.C. (2002): Cytochrome P450 pathways of arachidonic acid metabolism. Curr. Opin. Lipidol., 13, 273-283.

Lasker, J.M., Chen, W.B., Wolf, I., Bloswick, B.P., Wilson, P.D. and Powell, P.K. (2000): Formation of 20-hydroxyeicosatetraenoic acid, a vasoactive and natriuretic eicosanoid in human kidney. J. Biol. Chem., 275, 4118-4126.

Liu, H., Baliga, M. and Baliga, R. (2002): Effect of cytochrome P450 2E1 inhibitors on cisplatin-induced cytotoxicity to renal proximal tubular epithelial cells. Anticancer Res., 22, 863-868.

Liu, X.H., Li, J., Li, Q.X., Ai, Y.X. and Zhang, L. (2008): Protective effects of ligustrazine on cisplatin-induced oxidative stress, apoptosis and nephrotoxicity in rats. Environ. Toxicol. Pharmacol., 26, 49-55.

Lu, Y. and Cederbaum, A. (2007): The mode of cisplatin-induced cell death in CYP2E1-overexpressing HepG2 cells: modulation by ERK, ROS, glutathione, and thioredoxin. Free. Radic. Biol. Med., 43, 1061-1075.

Lu, Y. and Cederbaum, A.I. (2006): Cisplatin-induced hepatotoxicity is enhanced by elevated expression of cytochrome P450 2E1. Toxicol. Sci., 89, 515-523.

Merouani, A., Shpall, E.J., Jones, R.B., Archer, P.G. and Schrier, R.W. (1996): Renal function in high dose chemotherapy and autologous hematopoietic cell support treatment for breast cancer. Kidney Int., 50, 1026-1031.

Nakamura, M., Imaoka, S., Tanaka, E., Misawa, S. and Funae, Y. (1998): cis-Diamminedichloroplatinum induces peroxisomes as well as CYP4A1 in rat kidney. Res. Commun. Mol. Pathol. Pharmacol., 99, 23-32.

Portilla, D., Li, S., Nagothu, K.K., Megyesi, J., Kaissling, B.. Schnackenberg, L., Safirstein, R.L. and Beger, R.D. (2006): Metabolomic study of cisplatin-induced nephrotoxicity. Kidney Int., 69, 2194-2204.

Rahman, M., Wright, J.J. and Douglas, J. (1997): The role of the cytochrome P450-dependent metabolites of arachidonic acid in blood pressure regulation and renal Function: a review. Am. J. Hypertens., 10, 356-365.

Safirstein, R., Winston, J., Goldstein, M., Moel, D., Dikman, S. and Guttenplan, J. (1986): Cisplatin nephrotoxicity. Am. J. Kidney Dis., 8, 356-367.

Schrier, R.W., Wang, W., Poole, B. and Mitra, A. (2004): Acute renal failure: definitions, diagnosis, pathogenesis, and therapy. J. Clin. Invest., 114, 5-14.

Screnci, D. and McKeage, M.J. (1999): Platinum neurotoxicity: clinical profiles, experimental models and neuroprotective approaches. J. Inorg. Biochem., 77, 105-110.

Vickers, A.E., Rose, K., Fisher, R., Saulnier, M., Sahota, P. and Bentley, P. (2004): Kidney slices of human and rat to characterize cisplatin-induced injury on cellular pathways and morphology. Toxicol. Pathol., 32, 577-590.

Yao, X., Panichpisal, K., Kurtzman, N. and Nugent, K. (2007): Cisplatin nephrotoxicity: a review. Am. J. Med. Sci., 334, 115-124. 